\title{
Preface: DGAA Special Issue on Pursuit-Evasion Games and Differential Games with Incomplete Information
}

\author{
Valerii Patsko ${ }^{1}$ - Marc Quincampoix ${ }^{2}$ \\ Published online: 23 April 2019 \\ (c) Springer Science+Business Media, LLC, part of Springer Nature 2019
}

The differential game theory appeared in the middle of the twentieth century. Nowadays, in frames of zero-sum differential games when a motion is described by ordinary differential equations, it is possible to call "traditional" the following problems: questions of existence of the value function and investigation of its properties, establishing its correspondence with a solution of partial differential equation of the Hamilton-Jacobi type, numerical constructions of the value function and optimal strategies, solutions of model applied problems [1-4,9,11$15,20,22,23,25,26,28-30]$. In this scope, there are examples of the problem formulations and appropriate methods for their investigation. But till now, zero-sum differential games with many participants, problems that have incomplete (or uncertain) information about the current phase state of the controllable system, as well as problems where the motion is described by functional differential equations, are difficult and under-researched [5,10,16-19,24,27].

In recent years, new problems appeared, which deal with a motion of tremendous number of agents where each agent has a negligible effect but only the payoff is a function of the mean behaviour of all agents. A special term "mean field games" was invented for such games $[6,21]$. There are also new classes of differential games with an incomplete information of a probabilistic nature on the data (initial conditions, dynamics or payoffs) $[7,8]$.

This special issue of the Journal includes differential games of both the traditional and new directions.

In the article "Krasovskii-Subbotin approach to mean field type differential games" by Y. Averboukh, two antagonist players control a large number of agents that have the same dynamics given by a controlled ODE. It is assumed that the players are able to observe the distribution of agents. The author obtains the existence of the value function and gives some suboptimal strategies.

In the paper "Simultaneous multiple capture of rigidly coordinated evaders" by A.I. Blagodatskikh and N.N.Petrov, as well as in the paper "Capture of given number of

$\triangle$ Valerii Patsko

patsko@imm.uran.ru

Marc Quincampoix

marc.quincampoix@univ-brest.fr

1 N.N. Krasovskii Institute of Mathematics and Mechanics of the Ural Branch of the Russian Academy of Sciences (IMM UB RAS), 16 S.Kovalevskaya Str., Yekaterinburg, Russia 620108

2 Laboratoire de Mathématiques de Bretagne Atlantique, CNRS-UMR 6205, Université de Brest, 6 avenue Victor le Gorgeu, 29200 Brest, France 
evaders in Pontryagin's nonstationary example" by N.N. Petrov and N.A. Solov'eva, some formulations of differential games dealing with groups of pursuers and evaders are considered. Following the works by B. Pshenichnyi, A. Chikrii, and N. Grigorenko, the authors do not consider any criterion of optimality in the games. Only the capture fact is important. Sufficient (and in some cases, necessary) conditions under which the capture is possible are formulated.

The paper "Generation of dangerous disturbances for flight systems" by N.Botkin, K. Martynov, V.Turova, and J.Diepolder relates to the analysis of the worst wind disturbances in aviation problems, including the nonlinear problem of aircraft take-off. It uses the numerical method elaborated by the authors for solving linear differential games of high dimension.

In the work "Pursuit in the presence of a defender" by E. Garcia, D. Casbeer, and M.Pachter, a differential game of three players is considered. The Attacker pursues the Target, but the Defender shields the Target. Games of this type are called Attacker-TargetDefender (ATD) games and are intensively investigated now. In this work, the region of those initial positions in which the Attacker guarantees interception of the Target despite Defender's behaviour is investigated. For this region, an optimal strategy of the Attacker is found. The conjugation of this region with the area where the Defender hits the Attacker before the latter hits the Target is analysed.

The paper "Continuous-time Markov games with asymmetric information" by F. Gensbittel concerns antagonistic stochastic differential games with asymmetric information and with a payoff depending on a continuous-time Markov chain with finite state space that is observed only by the first player. The author obtains the existence of the value function and also its characterization as the unique solution of a suitable Hamilton-Jacobi equation with convex constraint.

The main peculiarity of differential games considered in the paper "On a solution of a guarantee optimization problem under the functional constraints on the disturbance" by M. Gomoyunov and D.Serkov is in the fact that, in addition to the geometric constraints imposed on the player controls, the maximizing player must obey a special functional constraint. Namely, its controls are taken from some unknown compact in $L_{1}$.

One of the most important particular problems in differential games is to build the solvability set of a conflict guidance onto a given target set at fixed terminal instant. Typical backward procedures are based on representation of the solvability set as a limit of those sets that are calculated on partitions of the time interval into subintervals getting smaller and smaller. The work "Computation of solvability set for differential games in the plane with simple motion and non-convex terminal set" by L. Kamneva proposes and proves an algorithm for constructing the solvability set in the games with polygonal and non-convex (in general) terminal sets and polygonal constraints on the controls. The algorithm gives the exact solution without using partitions of the time interval.

In the work "On time-optimal problems with lifeline" by S.S. Kumkov and N.V. Munts, a game with the "lifeline" is considered in a general formulation. The first player aspires to get the system motion onto a given terminal set. The second player is interested in getting the motion onto another terminal set (corresponding to his interests); the boundary of this set is called the "lifeline". Here, the principal questions are joined with the accurate formulation of the problem as a zero-sum differential game, ascertaining the properties of the value function, and determining its relation with the generalized solution of equation of the Hamilton-Jacobi type. These are the questions that comprise the paper subject.

The work "Non-stationary differential-difference games of neutral type" by I. Liubarshchuk, Y. Bihun, and I. Cherevko is devoted to a pursuit-evasion game where the dynamics is given 
by a system of linear functional differential equations of neutral type. For this problem, sufficient conditions ensuring the capture for any counteraction of the evader are provided.

The paper "Differential games on minmax of a positional quality index" by N. Lukoyanov and M. Gomoyunov is mainly an overview of the works by N.N. Krasovskii on differential games with the "positional functional". Motion of the system is linear, and the non-terminal quality index has certain convexity properties. A method of calculating the value function and optimal strategies of players is described. The implementation of optimal strategies is based only on some information about the current state of the system.

The paper "The classical homicidal chauffeur game" by M. Pachter and S. Coates is educational in nature. Here, the solution of the "homicidal chauffeur" game is considered in detail for one important case, which was described briefly in R. Isaacs' book "Differential Games" and in the dissertation by A. Merz.

Another version of the ATD problem is investigated in the work "Missile-Target-Defender problem with incomplete a priori information" by E. Rubinovich. Here, the Target using the minimal radius of its turn avoids the Attacker, which moves rectilinearly with the constant velocity. To defend himself from the Attacker, the Target uses special defending missile. It is necessary to calculate the Defender open-loop control such that the latter intercepts the Attacker under conditions when the line-of-sight Target-Attacker is known only at the initial time instant, but the Attacker velocity and the initial range of Target-Attacker are unknown.

The existence of the value of a zero-sum differential game with asymmetric information on the initial state and an infinite time horizon running payoff is investigated by $\mathrm{X}$. Wu in the article "Infinite horizon differential games with asymmetric information". At the beginning of the game, an unknown initial state is chosen randomly, and each player receives only a private signal generated by the initial state. The existence of the value function is obtained by the author together with its characterization as the unique solution of a suitable HamiltonJacobi-Isaacs equation.

Acknowledgements The guest editors would like to thank the referees who evaluated the submissions to this special issue. We also would like to thank the authors who have contributed to their works. We wish to thank Georges Zaccour, Editor-in-Chief of Dynamic Games and Applications, for proposing the special issue and for his continuous support.

\section{References}

1. Aubin J-P (1991) Viability theory. Birkäuser, Boston

2. Bardi M, Capuzzo-Dolcetta I (1997) Optimal control and viscosity solutions of Hamilton-Jacobi-Bellman equations. Birkhäuser, Boston

3. Başar T, Bernhard P (1995) H $\infty$-optimal control and related minimax design problems: a dynamic game approach. Birkhäuser, Basel

4. Başar T, Olsder GJ (1982) Dynamic noncooperative game theory. Academic Press, London

5. Bayraktar E, Keller C (2018) Path-dependent Hamilton-Jacobi equations in infinite dimensions. J Funct Anal 275:2096-2161

6. Bensoussan A, Frehse J, Yam P (2013) Mean field games and mean field type control theory. Springer, New York

7. Cardaliaguet P (2007) Differential games with asymmetric information. SIAM J Control Optim 46(3):816-838

8. Cardaliaguet P, Quincampoix M (2008) Deterministic differential games under probability knowledge of initial condition. Int Game Theory Rev 10(1):1-16

9. Cardaliaguet P, Quincampoix M, Saint-Pierre P (1999) Set-valued numerical analysis for optimal control and differential games. In: Bardi M, Raghavan TES, Parthasarathy T (eds) Stochastic and differential games. Annals of the international society of dynamic games, vol 4. Birkhäuser, Boston, pp 177-247

10. Chikrii A (1997) Conflict-controlled processes. Springer, Berlin 
11. Fleming WH (1961) The convergence problem for differential games. J Math Anal Appl 3:102-116

12. Friedman A (1971) Differential games. Wiley Interscience, New York

13. Isaacs R (1965) Differential games. Wiley, New York

14. Krasovskii AN, Krasovskii NN (1995) Control under lack of information. Birkhäuser, Boston

15. Krasovskii NN, Subbotin AI (1988) Game-theoretical control problems. Springer-Verlag, New York

16. Kryazhimskiy AV, Osipov YuS (2012) On the solvability of problems of guaranteeing control for partially observable linear dynamical systems. Proc Steklov Inst Math 277(1):144-159

17. Kumkov SS, Le Menec S, Patsko VS (2017) Zero-sum pursuit-evasion differential games with many objects: survey of publications. Dyn Games Appl 7(4):609-633

18. Kurzhanskii AB (2004) The problem of measurement feedback control. J Appl Math Mech 68(4):487-501

19. Kurzhanski AB, Varaiya P (2014) Dynamics and control of trajectory tubes. Birkhäuser, Basel

20. Lewin J (1994) Differential games. Springer, London

21. Lasry JM, Lions PL (2007) Mean field games. Jpn J Math 2(1):229-260

22. Melikyan AA (1998) Generalized characteristics of first order PDEs: application in optimal cintrol and differential games. Birkhäuser, Boston

23. Osipov YuS, Kryazhimskii AV (1995) Inverse problems for ordinary differential equations: dynamical solutions. Gordon and Breach Science Publishers, London

24. Pachter M, Garcia E, Casbeer DW (2019) Toward a solution of the active target defense differential game. Dyn Games Appl 9(1):165-216

25. Patsko V, Kumkov S, Turova V (2018) Pursuit-evasion games. In: Basar T, Zaccour G (eds) Handbook of dynamic game theory. Springer, Berlin, pp 951-1038

26. Petrosyan LA (1993) Differential games of pursuit. World Scientific, New York

27. Plaksin AR (2015) Finite-dimensional guides for conflict-controlled linear systems of neutral type. Differ Equ 48(3):406-416

28. Pontryagin LS (1981) Linear differential games of pursuit. Math USSR-Sbornik 40(3):285-303

29. Sethian JA (1999) Level set methods and fast marching methods. Cambridge University Press, Cambridge

30. Subbotin AI (1995) Generalized solutions of first-order PDEs. The dynamical perspective. Birkhäuser, Boston

Publisher's Note Springer Nature remains neutral with regard to jurisdictional claims in published maps and institutional affiliations. 